\section{Infection pattern of neutropenic patients in post-chemotherapy phase of acute leukemia treatment}

\author{
Ahmad Ahmadzadeh, ${ }^{1}$ \\ Mehran Varnasseri, ${ }^{2}$ \\ Mohammad Hossein Jalili, ${ }^{3}$ \\ Fatemeh Maniavi, ${ }^{4}$ Armita Valizadeh, ${ }^{5}$ \\ Mojtaba Mahmoodian, ${ }^{6}$ \\ Manouchehr Keyhani ${ }^{7}$ \\ ${ }^{1}$ Research Center of Thalassemia and \\ Hemoglobinopathy, Ahvaz Jundishapur \\ University of Medical Science, Ahvaz; \\ 2Department of Infectious Disease, Razi \\ Hospital, Ahvaz Jundishapur University \\ of Medical Science, Ahvaz; ${ }^{3}$ Department \\ of Medicine, Tehran University of Medical \\ Science, Tehran; ${ }^{4}$ Department of Infection \\ Control, Shafa Hospital, Ahvaz \\ Jundishapur University of Medical \\ Sciences, Ahvaz; ${ }^{5}$ Department of \\ Anatomy, Ahvaz Jundishapur University \\ of Medical Sciences, Ahvaz; ${ }^{6}$ Department \\ of Medicine, Ahvaz Jundishapur \\ University of Medical Sciences, Ahvaz; \\ ${ }^{7}$ Hematology and Oncology Research \\ Center, Vali Asr Hospital, Tehran \\ University of Medical Science, Tehran, \\ Iran
}

\section{Abstract}

Neutropenia following chemotherapy regimens in leukemia patients is of major concern since it makes these patients vulnerable to infections. If we can identify which germs are causing these infections, they can be annihilated or, at least, the most appropriate antibiotic therapy can be started immediately, even before we have the results of the culture. This retrospective multi-center study took place in 2012 and included patients with acute leukemia who had already undergone chemotherapy and who had been febrile for at least 16 hours. In order to assess the type of infection, different environments were chosen and the results were compared by t-test and $\chi^{2}$ tests. This study took place in four hospitals in Tehran and Ahwaz, Iran. The study population was made up of 89 patients: 37 with acute lymphoblastic leukemia and 52 with acute myeloid leukemia. The results revealed that blood was the most common site of infection. From all our positive cultures, it was seen that $85.4 \%$ of them had gram-negative bacteria with a dominance of $E$. coli of $25.8 \%$ over the other colonies. Also, antibiograms revealed the sensitivity of almost all the gram-negatives to amino glycosides. In contrast with most of the literature, in our patients, gram-negatives are the most common cause of infection and, therefore, administering amino glycosides would be the safest antibiotic therapy to prescribe before culture results are available.

\section{Introduction}

Acute leukemia is one of the most common malignancies worldwide. Chemotherapy and stem cell transplantation (HSCT) are the established therapeutic options for these patients. These methods cause neutropenia and carry, therefore, an increased risk of infections. ${ }^{1-3}$ Over recent years, significant progress in antibiotics has improved the outcome of leukemia treatments. ${ }^{4}$ Acute leukemia has two broad classifications: acute lymphoblastic leukemia (ALL) that is more common in younger (pediatric) patients, and acute myeloblastic leukemia (AML) that accounts for $20-25 \%$ of childhood leukemias and up to $80 \%$ of adult acute leukemias. ${ }^{5}$ Its association with infections, in spite of the progress made in the development of antibiotics, is still a major concern for healthcare professionals. On the other hand, assessment of the type of infection presented after chemotherapy plays a major role in achieving a better treatment outcome.

The epidemiological studies of infections in the area of treatment, for example, in hospitals, have identified the most common germs and helped us make a better prognosis after treatment of chemotherapy-associated infection. This is thanks to a correct prediction of the type of infection at the immediate onset of fever before culture results are available. On the contrary, late decision-making and wrong predictions can not only lead to the spread of infection, but can also result in failure of chemotherapy, delayed completion of remission, and poor treatment outcome.

In this study, we tried to identify the pattern of infections and their antibiotic resistance.

\section{Materials and Methods}

This is a multi-center retrospective study involving patients with leukemia admitted to the adult oncology departments of four hospitals in Tehran and Ahwaz, Iran. Inclusion criteria were: i) confirmed diagnosis of AML or ALL; ii) previous chemotherapy; iii) documented fever for at least $16 \mathrm{~h}$ (fever defined as a single body temperature reading by mouth of over $38.3^{\circ} \mathrm{C}$ or over $38^{\circ} \mathrm{C}$ for over $2 \mathrm{~h}$; iv) positive blood or other cultures (Table 1). A total of 89 patients fulfilled the inclusion criteria. Patients with other comorbidities such as HIV,
Correspondence: Ahmad Ahmadzadeh, Reaserch Center of Thalassemia and Hemoglobinopathy, Ahvaz Jundishapur University of Medical Science, Ahvaz, Iran.

Tel. +98.611.333.9092 - Fax: +98.611.333.5200

E-mail: ahmad.ahmadzadeh@gmail.com

Key words: acute leukemia, infection, antibiogram.

Contributions: the authors contributed equally.

Conflict of interests: the authors declare no potential conflict of interests.

Received for publication: 11 July 2013

Revision received: 8 October 2013

Accepted for publication:17 October 2013.

This work is licensed under a Creative Commons Attribution NonCommercial 3.0 License (CC BYNC 3.0).

(O) Copyright A. Ahmadzadeh et al., 2013

Licensee PAGEPress, Italy

Hematology Reports 2013; 5:e15

doi:10.4081/hr.2013.e15

rheumatoid disease or other infections were excluded from the study.

Patients had initially undergone a physical examination to rule out previous existence of infections and laboratory tests and imaging procedures to verify if there had been suspicious foci of infection. Patients with no infection sites received a chemotherapy regimen. After chemotherapy, patients were considered to be febrile if they had a single body temperature reading of over $38.3^{\circ} \mathrm{C}$ for more than $2 \mathrm{~h}$ (oral, axillary or tympanic reading). To determine the cause of the fever or the site of the infection, we reviewed all their workups, such as blood culture and smear, catheter culture, and chest X-ray. Two blood samples were collected from two separate veins for each patient during the first day of the fever. If any doubts remained about possible infection of the catheter or pharyngitis, another sample was obtained.

Samples had initially undergone gram staining for microscopic examinations and were cultured on blood agar, Mannitol salt agar (MSA) and McConkey agar for preliminary isolation. For fungi detection, the samples were cultured on saboroud agar (SC) and were subsequently identified according to the standard identification criteria. The colonies grown on McConkey agar were examined by standard biochemical tests for detecting gram-negative bacteria, and the colonies grown on MSA representing staphylococci were further double checked by standard biochemical tests, including catalase, coagulase and DNAse. ${ }^{6}$

Antibiotic susceptibility testing was performed by the Kirby-Bauer disc-diffusion 
method on Mueller Hinton agar (Hi Media, India) and the results were interpreted according to the current guidelines of the Clinical Laboratory Standards Institute (CLSI). ${ }^{7}$ Gramnegative bacteria were evaluated against amino glycosides, imipenem and ceftriaxone.

\section{Statistical analysis}

Data were analyzed using the SPSS software and numerical data were presented as mean \pm standard deviation (SD), median and range. Qualitative data were presented as numbers and percentages and the $\chi^{2}$ test was performed to examine the relation between qualitative variables. Comparison between numerical variables was performed using the t-test. $\mathrm{P}<0.05$ was considered statistically significant.

\section{Results}

The study was performed in four centers in Tehran and Ahwaz, Iran, during 2012. Among 89 selected patients, $42(47.2 \%)$ were male and 47 (52.8\%) were female with a male:female ratio of $0.89: 1$. Thirty-seven (41.6\%) of our patients had ALL and the remaining 52 (58.4\%) had AML. Of the cultures, $62.9 \%$ and $16.9 \%$ were drained from blood and urine, respectively. Another $10.1 \%$ were from sputum, $4.5 \%$ from nasal discharges, $1.1 \%$ from sinus abscesses, and $3.4 \%$ obtained from catheters.

After data analysis of the culture results, $E$ coli $(25.8 \%)$ and other gram-negative bacteria (20.02\%) were the most common germs. Entrobacter (18\%) and coagolase-negative staphylococcus (13.5\%) were the next prevalent germs. Candida (5.6\%), pseudomonas (4.5\%) and other germs (2.1\%) were found in the rest of the cultures. Of our 89 acute leukemia patients with fever and neutropenia, 37 had ALL. Of these, 4 (10.8\%) had gram-positive infection while $33(89.2 \%)$ were infected with gram-negatives. Of the 52 AML patients, 9 (17.3\%) had gram-positives and the others
(82.7\%) had gram-negatives. This shows that, in general terms, $85.4 \%$ of our patients were infected with gram-negative germs and only $14.6 \%$ of them had gram-positive bacteria.

In addition, from our 56 positive blood cultures, in $50(89.3 \%)$ of them we observed the growth of gram-negatives and just 6 of them showed gram-positive colonies. Also, of 15 positive urine cultures, $93.3 \%$ had gram-negative colonies. Among gram-positive colonies, 92.3\% were due to coagolase-negative staphylococcus, and among our gram-negative colonies, $30.3 \%$ were caused by $E$-coli and $21.1 \%$ by entrobacter colonies.

We also found that the most prevalent site of infection was the blood with $62.9 \%$ positive cultures. Our antibiograms showed the antibiotics we could use for the germs observed. For E-coli colonies, the most common colony in our cultures, antibiograms suggested amikacin and gentamicin to which they were very sensitive. E-coli colonies were also moderately sensitive to imipenem but totally resistant to ceftriaxone. Enterobacter colonies showed sensitivity to amikacin and gentamicin but, like $E$-coli, were only moderately sensitive to imipenem and only slightly sensitive to ceftriaxone (Table 2).

Table 1. Frequency of gram-negative and gram-positive bacterial infections in the study population.

\begin{tabular}{lccc} 
& Gram - & Gram + & Total \\
Patients & 76 & 13 & 89 \\
Acute lymphoblastic leukemia & 33 & 4 & 37 \\
\hline Acute myelooblastic leukemia & 43 & 9 & 52 \\
Cultures & & & \\
$\quad$ Blood & 50 & 6 & 56 \\
$\quad$ Urine cultures & 14 & 1 & 15 \\
\hline
\end{tabular}

Table 2. Frequency of gram-positive, gram-negative and candida bacterial infections and their sensitivity to treatment.

\begin{tabular}{|c|c|c|c|c|c|}
\hline Germ & Frequency & Percent & Aminoglycosides & Imipenem & Ceftriaxone \\
\hline $\begin{array}{l}\text { Gram positives } \\
\text { Staph A. } \\
\text { Coagulase neg. Staph } \\
\text { Gram positive cocci } \\
\text { Gram pos. bacilli }\end{array}$ & $\begin{array}{c}1 \\
12 \\
1 \\
18\end{array}$ & $\begin{array}{c}7.7 \\
92.3 \\
1.3 \\
23.7\end{array}$ & $\begin{array}{c}- \\
- \\
\text { Sensitive } \\
\text { Sensitive }\end{array}$ & $\begin{array}{c}- \\
- \\
\text { Moderate S. } \\
\text { Moderate S. }\end{array}$ & $\begin{array}{l}\text { NA } \\
\text { NA }\end{array}$ \\
\hline $\begin{array}{l}\text { Gram negatives } \\
\text { E-coli } \\
\text { Entrobacter } \\
\text { Gram neg. bacilli } \\
\text { Proteus } \\
\text { Pseudomonas } \\
\text { Klebsiella }\end{array}$ & $\begin{array}{l}23 \\
16 \\
1 \\
1 \\
4 \\
2\end{array}$ & $\begin{array}{c}30.3 \\
21.1 \\
1.3 \\
1.3 \\
5.3 \\
2.6\end{array}$ & $\begin{array}{l}\text { Sensitive } \\
\text { Sensitive } \\
\text { Sensitive } \\
\text { Sensitive } \\
\text { Sensitive } \\
\text { Sensitive }\end{array}$ & $\begin{array}{l}\text { Moderate S. } \\
\text { Moderate S. } \\
\text { Moderate S. } \\
\text { Moderate S. } \\
\text { Moderate S. } \\
\text { Moderate S. }\end{array}$ & $\begin{array}{c}\text { Resistant } \\
\text { Slightly S. } \\
\text { NA } \\
\text { NA } \\
\text { NA } \\
\text { NA }\end{array}$ \\
\hline Candida & 5 & 6.6 & Sensitive & Moderate S. & NA \\
\hline
\end{tabular}


Cairo University, Egypt, constituting $68.9 \%$ of organisms isolated over a period of six months. ${ }^{9}$ As a result of this survey, we established that the most prevalent bacterial infection among acute leukemia patients is due to gram-negatives. This is an important new finding since previous surveys had produced different results. This was due to various reasons. In previous studies, and also in some textbooks, staphylococcus, a gram-positive bacterium, is known to be the most prevalent germ to cause infection in neutropenic patients. Another prominent finding was the pattern of resistance. Gram-negative bacteria are more sensitive to amikacin, ceftriaxone and imipenem and show moderate sensitivity to ciprofloxacin. However, on the other hand, coagulase-negative staphylococcus was more sensitive to amikacin and gentamicin.

The identification of clinical or laboratory predictors of causes of morbidity and mortality in febrile patients with leukemia is crucial to early treatment. ${ }^{10}$ Given this, and also from our results, we suggest amino glycosides such as amikacin and gentamicin to be one of the firstline treatments of fever and bacteremia in post-chemotherapy bacterial infections in leukemia patients. Compared with carbapenem, amino glycosides are more potent and less expensive. Therefore, we suggest the use of amino glycosides as first-line treatment.

\section{Conclusions}

In conclusion, our results suggest that, in acute leukemia patients who have undergone chemotherapy and who now have fever and neutropenia, it should be assumed that this is a gram-negative bacterial infection and firstline treatment with amino glycosides should be started immediately. Once the antibiogram results from the cultures are available, the specific antibiotic can be administered. This would mean there is no need for further use of carbapenem in these patients, thus avoiding drug overuse and helping to prevent the emersion of resistant germs.

\section{References}

1. Cornely OA, Maertens J, Winston DJ, et al. Posaconazole vs. fluconazole or itraconazole prophylaxis in patients with neutropenia. N Engl J Med 2007;356:348-59.

2. Bow EJ, Laverdiere $M$, Lussier $\mathrm{N}$, et al. Antifungal prophylaxis for severely neutropenic chemotherapy recipients: a meta analysis of randomized- controlled clinical trials. Cancer 2002;94:3230-46.

3. Bucaneve G, Micozzi A, Menichetti F, et al. Levofloxacin to prevent bacterial infection in patients with cancer and neutropenia. $\mathrm{N}$
Engl J Med 2005;353:977-87.

4. Pizzo PA. Management of fever in patients with cancer and treatment-induced neutropenia. N Engl J Med 1993;328:1323-32.

5. Gurney JG, Bondy ML. Epidemiology of childhood cancer. In: Pizzo PA, Poplack DG, eds. Principles and practice of pediatric oncology. 5th Ed. Philadelphia: Lippincott Williams \& Wilkins; 2006. pp 1-13.

6. Forbes BA, Sahm DF, Weissfeld AS. Bailey and Scott's diagnostic microbiology. 12th Ed. St. Louis: Mosby Inc.; 2007. pp 389-397.

7. Clinical and Laboratory Standards Institute. Performance standards for antimicrobial susceptibility testing: seventeenth informational supplement. CLSI document M100-S17. Vol. 27, No 1. January 2007. Available from: http://www. microbiolab-bg.com/CLSI.pdf.

8. Hann I, Viscoli C, Paesmans M, et al. Comparison of outcome from febrile neutropenic episodes in children compared with adults: results from four EORTC studies. Br J Haematol 1997;99:580-8.

9. El-Mahallawy HA, Attia IA, Aly El-Din NH, Moneib N. Risk factor evaluation and prognosis of bacteremia in children with cancer. J Egypt Nat Cancer Inst 1999;11:299305.

10. Aledo A, Heller G, Ren L, et al. Septicemia and septic shock in pediatric patients: 140 consecutive cases on a pediatric hematology-oncology service. J Pediatr Hematol Oncol 1998;20:215-21. 